\title{
Boundedness of the multiple singular integral operators on product spaces*
}

\author{
Guoen Hu, Bolin MA and Qiyu Sun \\ (Received April 12, 1999; Revised October 27, 1999)
}

Abstract. In this paper, we consider the $L^{p}\left(\mathbb{R}^{m} \times \mathbb{R}^{n}\right)$ boundedness for the multiple singular integral operators of Fefferman type, defined by

$$
T f\left(x_{1}, x_{2}\right)=\text { p.v. } \int_{\mathbb{R}^{m} \times \mathbb{R}^{n}} h\left(\left|y_{1}\right|,\left|y_{2}\right|\right) \frac{\Omega\left(y_{1}^{\prime}, y_{2}^{\prime}\right)}{\left|y_{1}\right|^{m}\left|y_{2}\right|^{n}} f\left(x_{1}-y_{1}, x_{2}-y_{2}\right) d y_{1} d y_{2},
$$

where $y_{1} \in \mathbb{R}^{m}, y_{2} \in \mathbb{R}^{n}$ and $y_{i}^{\prime}=y_{i} /\left|y_{i}\right|, h(r, s)$ is bounded on $\mathbb{R}_{+} \times \mathbb{R}_{+}, \Omega$ satisfies the cancellation condition

$$
\int_{S^{m-1}} \Omega\left(y_{1}^{\prime}, y_{2}^{\prime}\right) d y_{1}^{\prime}=\int_{S^{n-1}} \Omega\left(y_{1}^{\prime}, y_{2}^{\prime}\right) d y_{2}^{\prime}=0
$$

We show that if $\Omega \in L\left(\log ^{+} L\right)^{2}\left(S^{m-1} \times S^{n-1}\right)$, then $T$ is bounded on $L^{p}\left(\mathbb{R}^{m} \times \mathbb{R}^{n}\right)$ for all $1<p<\infty$.

Key words: multiple singular integral operator, Fourier transform estimate, LittlewoodPaley theory.

\section{Introduction and Statement of the Result}

Let $h(r, s)$ be a bounded function on $\mathbb{R}_{+} \times \mathbb{R}_{+}$, and $\Omega\left(y_{1}, y_{2}\right)$ a function defined on $S^{m-1} \times S^{n-1}(m, n \geq 2)$ satisfying

$$
\int_{S^{m-1}} \Omega\left(y_{1}^{\prime}, y_{2}^{\prime}\right) d y_{1}^{\prime}=\int_{S^{n-1}} \Omega\left(y_{1}^{\prime}, y_{2}^{\prime}\right) d y_{2}^{\prime}=0
$$

where $S^{m-1}$ (resp. $S^{n-1}$ ) is the unit sphere of $\mathbb{R}^{n}$ (resp. $\mathbb{R}^{m}$ ). For $y \in \mathbb{R}^{m}$, let $y^{\prime}=y /|y|$. Define the multiple singular integral operator

$$
\begin{aligned}
& T f\left(x_{1}, x_{2}\right) \\
& =\text { p.v. } \int_{\mathbb{R}^{m} \times \mathbb{R}^{n}} h\left(\left|y_{1}\right|,\left|y_{2}\right|\right) \frac{\Omega\left(y_{1}^{\prime}, y_{2}^{\prime}\right)}{\left|y_{1}\right|^{m}\left|y_{2}\right|^{n}} f\left(x_{1}-y_{1}, x_{2}-y_{2}\right) d y_{1} d y_{2} .
\end{aligned}
$$

1991 Mathematics Subject Classification : 42B20.

*Supported by the NSF of China $(19701039,19971010)$ and the Hunan Provincial Sciences foundation of China. 
Operators of this type have been considered by many authors. For the special case of $h(r, s)=1$, R. Fefferman [2] proved that if $\Omega$ satisfies appropriate regularity condition, then $T$ is bounded on $L^{p}\left(\mathbb{R}^{m} \times \mathbb{R}^{n}\right)$ for all $1<p<\infty$. By Fourier transform estimate and the Littlewood-Paley estimate, Duoandikoetxea [1] showed that for $\Omega \in L^{q}\left(\mathbb{R}^{m} \times \mathbb{R}^{n}\right), T$ is a bounded operator on $L^{p}\left(\mathbb{R}^{m} \times \mathbb{R}^{n}\right)$ for $1<p<\infty$. In this paper, we give a weeker condition than others under which the bounded result on $L^{p}$ would hold. Our statement is as following.

Theorem 1 Let $\Omega$ and $h$ be the same as above. Suppose that $\Omega$ belongs to the space $L\left(\log ^{+} L\right)^{2}\left(S^{m-1} \times S^{n-1}\right)$, then the operator $T$ defined by (2) is bounded on $L^{p}\left(\mathbb{R}^{m} \times \mathbb{R}^{n}\right)$ for all $1<p<\infty$.

\section{Proof of Theorem 1}

We begin with some preliminary lemmas.

Lemma 1 Let $\Omega\left(y_{1}^{\prime}, y_{2}^{\prime}\right)$ be integrable on $S^{m-1} \times S^{n-1}$. Then the maximal operator

$$
\begin{aligned}
& M_{\Omega} f\left(x_{1}, x_{2}\right) \\
& =\sup _{r, s>0} r^{-m} s^{-n}\left|\int_{\left|y_{1}\right|<r,\left|y_{2}\right|<s} \frac{\Omega\left(y_{1}^{\prime}, y_{2}^{\prime}\right)}{\left|y_{1}\right|^{m}\left|y_{2}\right|^{n}} f\left(x_{1}-y_{1}, x_{2}-y_{2}\right) d y_{1} d y_{2}\right|
\end{aligned}
$$

is bounded on $L^{p}\left(\mathbb{R}^{m} \times \mathbb{R}^{n}\right)$ for all $1<p<\infty$ with bound $C_{n, m, p}\|\Omega\|_{1}$.

This Lemma can be proved by the standard method of rotation of Calderón and Zygmund, see also [2, p. 885].

Lemma 2 Let $\left\{\sigma_{u, v}\right\}_{u, v \in \mathbb{Z}}$ be a sequence of Borel measures on $\mathbb{R}^{m} \times \mathbb{R}^{n}$ such that $\left\|\sigma_{u, v}\right\| \leq 1$. Suppose that the maximal operator

$$
\sigma^{*} f\left(x_{1}, x_{2}\right)=\sup _{u, v}|| \sigma_{u, v}\left|* f\left(x_{1}, x_{2}\right)\right|
$$

is bounded on $L^{p_{0}}\left(\mathbb{R}^{m} \times \mathbb{R}^{n}\right)$ for some $p_{0}$ with $1<p_{0}<\infty$. Then the inequality

$$
\left\|\left(\sum_{u, v}\left|\sigma_{u, v} * g_{u, v}\right|^{2}\right)^{1 / 2}\right\|_{p} \leq C\left\|\left(\sum_{u, v}\left|g_{u, v}\right|^{2}\right)^{1 / 2}\right\|_{p}
$$

holds for all $p$ with $\left|\frac{1}{p}-\frac{1}{2}\right| \leq \frac{1}{2 p_{0}}$. 
For the proof of Lemma 2, the readers see the analogous result in [1, p.198].

Lemma 3 Let $\Omega$ be a bounded function on $S^{m-1} \times S^{n-1}$. For $u, v \in \mathbb{Z}$, denote

$$
\begin{aligned}
& K_{u, v}\left(x_{1}, x_{2}\right) \\
& =\left|x_{1}\right|^{-m}\left|x_{2}\right|^{-n} h\left(\left|x_{1}\right|,\left|x_{2}\right|\right) \Omega\left(x_{1}^{\prime}, x_{2}^{\prime}\right) \chi_{\left\{2^{u} \leq\left|x_{1}\right| \leq 2^{u+1}, 2^{v}<\left|x_{2}\right| \leq 2^{v+1}\right\}}\left(x_{1}, x_{2}\right) .
\end{aligned}
$$

Then there exist positive constants $C$ and $\varepsilon$ which are independent of $\Omega, u$ and $v$, such that for $\xi_{1} \in \mathbb{R}^{m}, \xi_{2} \in \mathbb{R}^{n},\left|\xi_{1}\right|,\left|\xi_{2}\right| \neq 0$,

$$
\left|\widehat{K_{u, v}}\left(\xi_{1}, \xi_{2}\right)\right| \leq C\|\Omega\|_{\infty}\left(\left|2^{u} \xi_{1}\right|\left|2^{v} \xi_{2}\right|\right)^{-\varepsilon}
$$

On the other hand, if $\Omega$ is integrable on $S^{m-1} \times S^{n-1}$ and satisfies the cancellation condition (1), then

$$
\left|\widehat{K_{u, v}}\left(\xi_{1}, \xi_{2}\right)\right| \leq C\|\Omega\|_{1}\left|2^{u} \xi_{1}\right|\left|2^{v} \xi_{2}\right|,
$$

where $\widehat{K_{u, v}}$ is the Fourier transform of $K_{u, v}$.

The proof of Lemma 3 is implied in [1, p.193-194].

Remark Let $\sigma_{u, v}=K_{u, v}\left(x_{1}, x_{2}\right)$ in Lamma 2. In the situation, by the estimates in (3) and (4), following the same proofs as in [3], we can prove the maximal operator $\sigma^{*}$ is bounded on $L^{2}$. By boot-strap method, we can obtain that the maximal operator $\sigma^{*}$ is bounded on $L^{p}$ for $1<p<\infty$ and Lemma 2 holds for all $1<p<\infty$.

Proof of Theorem 1. Let $\phi^{1}$ and $\phi^{2}$ be two Schwarz functions on $\mathbb{R}^{m}$ and $\mathbb{R}^{n}$ respectively, such that

(a) $0 \leq \phi^{1}, \phi^{2} \leq 1, \operatorname{supp} \phi^{1} \subset\left\{x \in \mathbb{R}^{m}, 1 / 2 \leq|x| \leq 2\right\}$, supp $\phi^{2} \subset\{y \in$ $\left.\mathbb{R}^{n}, 1 / 2 \leq|y| \leq 2\right\}$

(b) $\sum_{k=-\infty}^{\infty} \phi^{1}\left(2^{k} x\right)^{2}=\sum_{l=-\infty}^{\infty} \phi^{2}\left(2^{l} y\right)^{2}=1$ for $x \in \mathbb{R}^{m}, y \in \mathbb{R}^{n}$ such that $|x|,|y| \neq 0$.

Set $\phi_{k}^{1}(x)=\phi^{1}\left(2^{k} x\right)$ and $\phi_{l}^{2}(y)=\phi^{2}\left(2^{l} y\right)$. Define the operators $S_{k}^{1}$ in $\mathbb{R}^{m}$ and $S_{l}^{2}$ in $\mathbb{R}^{n}$ by

$$
\widehat{S_{k}^{1} f}\left(\xi_{1}\right)=\phi_{k}^{1}\left(\xi_{1}\right) \hat{f}\left(\xi_{1}\right), \quad \widehat{S_{l}^{2} h}\left(\xi_{2}\right)=\phi_{l}^{2}\left(\xi_{2}\right) \hat{h}\left(\xi_{2}\right)
$$


and $S_{k}^{1} \otimes S_{l}^{2}$ in $\mathbb{R}^{m} \times \mathbb{R}^{n}$ by

$$
\left(S_{k}^{1 \otimes S_{l}^{2}} f\right)\left(\xi_{1}, \xi_{2}\right)=\phi_{k}^{1}\left(\xi_{1}\right) \phi_{l}^{2}\left(\xi_{2}\right) \widehat{f}\left(\xi_{1}, \xi_{2}\right)
$$

For fixed $k, l \in \mathbb{Z}$ and $\sigma_{u, v}$ as in the remark as above, denote by $U_{k, l}$ the operator defined by

$$
U_{k, l} f\left(x_{1}, x_{2}\right)=\sum_{u, v} S_{u-k}^{1} \otimes S_{v-l}^{2} \sigma_{u, v} *\left(\left(S_{u-k}^{1} \otimes S_{v-l}^{2}\right) f\right)\left(x_{1}, x_{2}\right) .
$$

Lemma 1 and Remark via the Littlewood-Paley theory (see [5]) state that

$$
\left\|U_{k, l} f\right\|_{p} \leq C\|\Omega\|_{1}\|f\|_{p}, \quad 1<p<\infty
$$

Decompose the operator $T$ as

$$
\begin{array}{rl}
T & f\left(x_{1}, x_{2}\right) \\
= & \sum_{k, l} \sum_{u, v} S_{u-k}^{1} \otimes S_{v-l}^{2} \sigma_{i, j} *\left(\left(S_{u-k}^{1} \otimes S_{v-l}^{2}\right) f\right)\left(x_{1}, x_{2}\right) \\
= & \sum_{k, l \leq 0} U_{k, l} f\left(x_{1}, x_{2}\right)+\sum_{k \leq 0, l>0} U_{k, l} f\left(x_{1}, x_{2}\right) \\
& \quad+\sum_{k>0, l \leq 0} U_{k, l} f\left(x_{1}, x_{2}\right)+\sum_{k>0, l>0} U_{k, l} f\left(x_{1}, x_{2}\right) \\
= & T_{\mathrm{I}} f\left(x_{1}, x_{2}\right)+T_{\mathrm{II}} f\left(x_{1}, x_{2}\right)+T_{\mathrm{III}} f\left(x_{1}, x_{2}\right)+T_{\mathrm{IV}} f\left(x_{1}, x_{2}\right) .
\end{array}
$$

By (5) together with Plancherel's theorem we see that

$$
\begin{aligned}
& \left\|U_{k, l} f\right\|_{2}^{2} \\
& \leq C \sum_{u, v=-\infty}^{\infty}\left\|\sigma_{u, v} *\left(\left(S_{u-k}^{1} \otimes S_{v-l}^{2}\right) f\right)\right\|_{2}^{2} \\
& =\sum_{u, v=-\infty}^{\infty} \int_{\mathbb{R}^{m} \times \mathbb{R}^{n}}\left|\widehat{\sigma_{u, v}}\left(\xi_{1}, \xi_{2}\right) \hat{f}\left(\xi_{1}, \xi_{2}\right) \phi^{1}\left(2^{u-k} \xi_{1}\right) \phi^{2}\left(2^{v-l} \xi_{2}\right)\right|^{2} d \xi_{1} d \xi_{2} \\
& \leq C\left(2^{k} 2^{l}\right)^{2} \sum_{u, v=-\infty}^{\infty}\left\|\left(S_{u-k}^{1} \otimes S_{v-l}^{2}\right) f\right\|_{2}^{2} \\
& \leq C\left(2^{k} 2^{l}\right)^{2}\|f\|_{2}^{2} .
\end{aligned}
$$

Interpolation between the inequalities (5) and (6) shows that for $1<p<\infty$, 
there exists a positive constant $\delta=\delta_{p}>0$ such that

$$
\left\|U_{k, l} f\right\|_{p} \leq C 2^{\delta l} 2^{\delta k}\|f\|_{p} .
$$

This in turn leads to the estimate

$$
\left\|T_{\mathrm{I}} f\right\|_{p} \leq C\|f\|_{p} \sum_{k<0} 2^{\delta k} \sum_{l<0} 2^{\delta l} \leq C\|f\|_{p}, \quad 1<p<\infty .
$$

Now we turn our attention to $T_{\mathrm{IV}}$. Let $E_{0}=\left\{\left(x_{1}^{\prime}, x_{2}^{\prime}\right) \in S^{m-1} \times S^{n-1}\right.$, $\left.\left|\Omega\left(x_{1}^{\prime}, x_{2}^{\prime}\right)\right| \leq 1\right\}$ and $E_{d}=\left\{\left(x_{1}^{\prime}, x_{2}^{\prime}\right) \in S^{m-1} \times S^{n-1}, 2^{d-1}<\left|\Omega\left(x_{1}^{\prime}, x_{2}^{\prime}\right)\right| \leq\right.$ $\left.2^{d}\right\}$ for positive integer $d$. Denote by $\Omega_{d}$ the restriction of $\Omega$ on $E_{d}$. Our assumption implies that $\sum_{d>0} d^{2} 2^{d}\left|E_{d}\right|<\infty$. Set

$$
\begin{aligned}
& \sigma_{u, v}^{d}\left(y_{1}, y_{2}\right) \\
& =h\left(\left|y_{1}\right|,\left|y_{2}\right|\right)\left|y_{1}\right|^{-m}\left|y_{2}\right|^{-n} \Omega_{d}\left(y_{1}, y_{2}\right) \chi_{\left\{2^{u}<\left|y_{1}\right| \leq 2^{u+1}, 2^{v}<\left|y_{2}\right| \leq 2^{v+1}\right\}}\left(y_{1}, y_{2}\right),
\end{aligned}
$$

and $U_{k, l}^{d}$ defined in the same way as that in the definition of $U_{k, l}$, but with $\sigma_{u, v}$ replacing by $\sigma_{u, v}^{d}$. Again by Lemma 1 and Lemma 2,

$$
\left\|U_{k, l}^{d} f\right\|_{p} \leq C\left\|\Omega_{d}\right\|_{1}\|f\|_{p}, \quad 1<p<\infty .
$$

Let $N$ be a integer and $N>2 \varepsilon^{-1}$, where $\varepsilon$ is the positive constant in Lemma 3. Write

$$
\begin{array}{rl}
T_{\mathrm{IV}} & f\left(x_{1}, x_{2}\right) \\
= & \sum_{l>0} \sum_{k>0} U_{k, l}^{0} f\left(x_{1}, x_{2}\right)+\sum_{d>0} \sum_{0<k \leq N d} \sum_{0<l \leq N d} U_{k, l}^{d} f\left(x_{1}, x_{2}\right) \\
& \quad+\sum_{d>0} \sum_{0<k \leq N d l>N d} \sum_{k, l} U_{k}^{d} f\left(x_{1}, x_{2}\right)+\sum_{d>0} \sum_{k>N d} \sum_{l>0} U_{k, l}^{d} f\left(x_{1}, x_{2}\right) \\
= & T_{\mathrm{IV}}^{0} f\left(x_{1}, x_{2}\right)+T_{\mathrm{IV}}^{1} f\left(x_{1}, x_{2}\right)+T_{\mathrm{IV}}^{2} f\left(x_{1}, x_{2}\right)+T_{\mathrm{IV}}^{3} f\left(x_{1}, x_{2}\right)
\end{array}
$$

It follows from Lemma 3 that

$$
\begin{aligned}
& \left\|U_{k, l}^{d} f\right\|_{2}^{2} \\
& \leq C \sum_{u, v=-\infty}^{\infty} \int_{\mathbb{R}^{m} \times \mathbb{R}^{n}}\left|\widehat{\sigma_{u, v}^{d}}\left(\xi_{1}, \xi_{2}\right) \hat{f}\left(\xi_{1}, \xi_{2}\right) \phi^{1}\left(2^{u-k} \xi_{1}\right) \phi^{2}\left(2^{v-l} \xi_{2}\right)\right|^{2} d \xi_{1} d \xi_{2} \\
& \leq C\left(\left\|\Omega_{d}\right\|_{\infty} 2^{-\varepsilon k} 2^{-\varepsilon l}\right)^{2} \sum_{u, v=-\infty}^{\infty}\left\|\left(S_{u-k}^{1} \otimes S_{v-l}^{2}\right) f\right\|_{2}^{2} \\
& \leq C\left(\left\|\Omega_{d}\right\|_{\infty} 2^{-\varepsilon k} 2^{-\varepsilon l}\right)^{2}\|f\|_{2}^{2} .
\end{aligned}
$$


Combining the estimate (7) and (8) we thus have that for $1<p<\infty$,

$$
\left\|U_{k, l}^{d} f\right\|_{p} \leq C\left\|\Omega^{d}\right\|_{\infty}^{t} 2^{-t \varepsilon k} 2^{-t \varepsilon l}\|f\|_{p}
$$

where $0<t<1$ is a constant depending only on $p$. So,

$$
\left\|T_{\mathrm{IV}}^{0} f\right\|_{p} \leq C \sum_{k, l>0} 2^{-t \varepsilon k} 2^{-t \varepsilon l}\|f\|_{p} \leq C\|f\|_{p}, \quad 1<p<\infty .
$$

Similarly, we have

$$
\begin{aligned}
\left\|T_{\mathrm{IV}}^{2} f\right\|_{p} & \leq C \sum_{d>0} \sum_{0<k \leq N d, l>N d}\left\|U_{k, l}^{d}\right\|_{p} \\
& \leq C \sum_{d>0} 2^{t d} \sum_{0<k<N d, l>N d} 2^{-t \varepsilon k} 2^{-t \varepsilon l}\|f\|_{p} \\
& \leq C \sum_{d>0} 2^{t d} 2^{-t \varepsilon N d}\|f\|_{p} \leq C\|f\|_{p},
\end{aligned}
$$

and

$$
\left\|T_{\mathrm{IV}}^{3} f\right\| \leq C\|f\|_{p}
$$

On the other hand, it is easy to see that

$$
\begin{aligned}
\left\|T_{\mathrm{IV}}^{1} f\right\|_{p} & \leq C \sum_{d>0}\left\|\Omega_{d}\right\|_{1} \sum_{0<k<N d} \sum_{0<l<N d}\|f\|_{p} \\
& \leq C \sum_{d>0} d^{2} 2^{d}\left|E_{d}\right|\|f\|_{p} \leq C\|f\|_{p} .
\end{aligned}
$$

It remains to estimate $T_{\mathrm{II}}$ and $T_{\mathrm{III}}$. We only consider $T_{\mathrm{II}}$, the other can be treated in the same way. Let $\widetilde{\Omega}\left(x_{2}^{\prime}\right)=\left\|\Omega\left(\cdot, x_{2}^{\prime}\right)\right\|_{L^{1}\left(S^{m-1}\right)}$. Set $\widetilde{E_{0}}=\left\{x_{2}^{\prime} \in\right.$ $\left.S^{n-1}, \widetilde{\Omega}\left(x_{2}^{\prime}\right) \leq 1\right\}$, and $\widetilde{E_{d}}=\left\{x_{2}^{\prime} \in S^{n-1}, 2^{d-1}<\widetilde{\Omega}\left(x_{2}^{\prime}\right) \leq 2^{d}\right\}$ for positive integer $d$. By Jensen's inequality, we see that $\widetilde{\Omega} \in L\left(\log ^{+} L\right)^{2}\left(S^{n-1}\right)$ and so $\sum_{d>0} d^{2} 2^{d}\left|\widetilde{E_{d}}\right|<+\infty$. Denote by $\widetilde{\Omega_{d}}$ the restriction of $\Omega$ on $S^{m-1} \times \widetilde{E_{d}}$. Let

$$
\begin{aligned}
\widetilde{\sigma_{u, v}^{d}}\left(y_{1}, y_{2}\right)=h\left(\left|y_{1}\right|,\left|y_{2}\right|\right)\left|y_{1}\right|^{-m}\left|y_{2}\right|^{-n} \widetilde{\Omega_{d}}\left(y_{1}, y_{2}\right) \\
\\
\chi_{\left\{2^{u}<\left|y_{1}\right| \leq 2^{u+1}, 2^{v}<\left|y_{2}\right| \leq 2^{v+1}\right\}}\left(y_{1}, y_{2}\right),
\end{aligned}
$$

and $\widetilde{U_{k, l}^{d}}$ be defined in the same way as that in the definition of $U_{k, l}$, but 
with $\sigma_{u, v}$ replacing by $\widetilde{\sigma_{u, v}^{d}}$. We claim that

$$
\left|\widehat{\widehat{\sigma_{u, v}^{d}}}\left(\xi_{1}, \xi_{2}\right)\right| \leq C \min \left\{2^{d}\left|2^{u} \xi_{1}\right|\left|2^{v} \xi_{2}\right|^{-\varepsilon}, 2^{d}\left|\widetilde{E_{d}}\right|\left|2^{u} \xi_{1}\right|\right\}
$$

In fact, the estimate

$$
\left|\widehat{\widetilde{\sigma_{u, v}^{d}}}\left(\xi_{1}, \xi_{2}\right)\right| \leq C 2^{d}\left|\widetilde{E_{d}}\right|\left|2^{u} \xi_{1}\right|
$$

follows from the cancellation property of $\Omega\left(x_{1}, x_{2}\right)$ on $x_{1}$. Write

$$
\begin{aligned}
& \left|\widehat{\widetilde{\sigma_{u, v}^{d}}}\left(\xi_{1}, \xi_{2}\right)\right| \leq C \int_{2^{v}}^{2^{v+1}} \int_{2^{u}}^{2^{u+1}} \\
& \left|\int_{S^{n-1}} e^{i s \xi_{2} x_{2}^{\prime}} \int_{S^{m-1}}\left(e^{i r \xi_{1} x_{1}^{\prime}}-1\right) \widetilde{\Omega_{d}}\left(x_{1}^{\prime}, x_{2}^{\prime}\right) d x_{1}^{\prime} d x_{2}^{\prime}\right| \frac{d r}{r} \frac{d s}{s} .
\end{aligned}
$$

For each fixed $r, \xi_{1}$, set

$$
\Omega_{d ; r, \xi_{1}}\left(x_{2}^{\prime}\right)=\int_{S^{m-1}}\left(e^{i r \xi_{1} x_{1}}-1\right) \widetilde{\Omega_{d}}\left(x_{1}^{\prime}, x_{2}^{\prime}\right) d x_{1}^{\prime} .
$$

A well-known result of Duoandikoetxea and Rubio de Francia [2] shows that

$$
\begin{aligned}
\int_{2^{v}}^{2^{v}+1} & \left|\int_{S^{n-1}} e^{s \xi_{2} x_{2}^{\prime}} \int_{S^{m-1}}\left(e^{i r \xi_{1} x_{1}}-1\right) \widetilde{\Omega_{d}}\left(x_{1}^{\prime}, x_{2}^{\prime}\right) d x_{1}^{\prime} d x_{2}^{\prime}\right| \frac{d s}{s} \\
& \leq C\left|2^{v} \xi_{2}\right|^{-\varepsilon}\left\|\Omega_{d ; r, \xi_{1}}\right\|_{L^{\infty}\left(S^{n-1}\right)} \\
& \leq C\left|2^{v} \xi_{2}\right|^{-\varepsilon}\left|r \xi_{1}\right|\left\|\int_{S^{m-1}}\left|\widetilde{\Omega_{d}}\left(x_{1}^{\prime}, x_{2}^{\prime}\right)\right| d x_{1}^{\prime}\right\|_{L^{\infty}\left(S^{n-1}\right)} \\
& \leq C 2^{d}\left|2^{v} \xi_{2}\right|^{-\varepsilon}\left|r \xi_{1}\right|
\end{aligned}
$$

Straightforward computation then establishes our claim. Plancherel's theorem now tells us that

$$
\left\|\widetilde{U_{k, l}^{d}} f\right\|_{2} \leq C \min \left\{2^{d} 2^{k} 2^{-\varepsilon l}, 2^{d}\left|\widetilde{E_{d}}\right| 2^{k}\right\}\|f\|_{2} .
$$

On the other hand, We know that

$$
\left\|\widetilde{U_{k, l}^{d}} f\right\|_{p} \leq C\left\|\widetilde{\Omega_{d}}\right\|_{1}\|f\|_{p} \leq C 2^{d}\left|\widetilde{E_{d}}\right|\|f\|_{p}, \quad 1<p<\infty .
$$


It follows from the last two inequalities that for each $1<p<\infty$,

$$
\begin{aligned}
\left\|\widetilde{U_{k, l}^{d}} f\right\|_{p} & \leq C \min \left\{2^{d}\left|\widetilde{E_{d}}\right|^{1-t} 2^{t k} 2^{-t \varepsilon l}, 2^{t k} 2^{d}\left|\widetilde{E_{d}}\right|\right\}\|f\|_{p} \\
& \leq C \min \left\{2^{t k} 2^{t d} 2^{-\varepsilon t l}, 2^{t k} 2^{d}\left|\widetilde{E_{d}}\right|\right\}\|f\|_{p},
\end{aligned}
$$

with $t=t_{p} \in(0,1)$ (note that $\left.2^{d}\left|\widetilde{E_{d}}\right| \leq C\right)$. Write

$$
\begin{aligned}
T_{\mathrm{II}} f\left(x_{1}, x_{2}\right)=\sum_{k \leq 0} & \sum_{l>0} \widetilde{U_{k, l}^{0}} f\left(x_{1}, x_{2}\right)+\sum_{k \leq 0} \sum_{d>0} \sum_{0<l \leq N d} \widetilde{U_{l, k}^{d}} f\left(x_{1}, x_{2}\right) \\
& +\sum_{k \leq 0} \sum_{d>0} \sum_{l>N d} \widetilde{U_{l, k}^{d}} f\left(x_{1}, x_{2}\right) .
\end{aligned}
$$

Therefore,

$$
\sum_{k \leq 0} \sum_{l>0}\left\|\widetilde{U_{k, l}^{0}} f\right\|_{p} \leq C \sum_{k \leq 0} \sum_{l>0} 2^{t k} 2^{-t \varepsilon l}\|f\|_{p} \leq C\|f\|_{p}
$$

and

$$
\sum_{k \leq 0} \sum_{d>0} \sum_{l>N d}\left\|\widetilde{U_{l, k}^{d}} f\right\|_{p} \leq C\|f\|_{p} \sum_{k \leq 0} 2^{t k} \sum_{d>0} 2^{t d} \sum_{l>N d} 2^{-t \varepsilon l} \leq C\|f\|_{p}
$$

Finally, we have,

$$
\sum_{k \leq 0} \sum_{d>0} \sum_{0<l \leq N d}\left\|\widetilde{U_{l, k}^{d}} f\right\|_{p} \leq C\|f\|_{p} \sum_{k \leq 0} 2^{t k} \sum_{d>0} 2^{d} d\left|\widetilde{E_{d}}\right| \leq C\|f\|_{p}
$$

This completes the proof of Theorem 1 .

\section{References}

[1] Duoandikoetxea J., Multiple singular integrals and maximal functions along hypersurfaces. Ann. Inst Fourier Grenoble 36 (1986), 185-206.

[2] Duoandikoetxea J., Weighted norm inequalities for homogeneous singular integrals. Trans Amer. Math. Soc. 336 (1993), 869-886.

[3] Duoandikoetxea J. and Rubio de Francia J.L., Maximal and singular integral operators via Fourier transform estimatess. Invent. Math. 84 (1986), 541-561.

[4] Fefferman R., Singular integrals on product domains. Bull. Amer. Math. Soc. 4 (1981), 195-201.

[5] Stein E.M., Singular Integrals and Differentiablity Properties of Functions, Princeton Univ. Press, Princeton, New Jersey, 1970. 
Guoen $\mathrm{Hu}$

Department of Applied Mathematics Institute of Information

P. O. Box 1007-47 Zhengzhou

People's Republic of China

Bolin Ma

Department of Applied Mathematics

Hunan University

Changsha, Hunan 410082

People's Republic of China

Qiyu Sun

Center for Mathematical Science Zhejiang University

Hangzhou, Zhejiang 310027

Peopel's Republic of China 